\title{
Investigation of adsorption and desorption behavior of small-volume cylinders and its relevance for atmospheric trace gas analysis
}

Ece Satar et al.

Correspondence to: Ece Satar (ece.satar@climate.unibe.ch)

The copyright of individual parts of the supplement might differ from the CC BY 4.0 License. 


\section{S1 Supplementary material on analysis of CRDS data}

In order to find the best strategy for the data interpretation and a suitable cut-off point presented in Sect. 3.1.1, three methods were applied.

(i) using $\mathrm{CO}$ measurements and set the end point when the $\mathrm{CO}$ amount fraction starts to increase

(ii) setting a minimum gas inflow to the analyzer limiting the residence time in the cavity.

(iii) applying a method using the correlation between an internal variable of the analyzer (outlet valve) and measured variables (low pressure reading of the pressure regulator).

Since (ii) is already explained in Sect. 3.1.1, the details of the two other methods are presented here.

\section{S1.1 Using CO measurements}

The method is based on the assumption that the measurements of CO should not show an increase due to adsorption/desorption processes. This is a reasonable assumption considering previous experiments with big cylinders (unpublished data).

For each of the gas species, the deviation from the mean of the first hour of measurements was calculated, and then this was divided by the maximum of the calculated differences to obtain the normalized species (Fig. S1). For the low pressure side of the pressure regulator, the difference was calculated relative to the highest pressure, whereas for the outlet valve the minimum value of 17500 was taken. A similar strategy to time of emergence (ToE), used in climate sciences to distinguish natural variability from climate change (Hawkins and Sutton, 2012), was adopted to separate instrumental noise of the CO measurements from the increasing signal. Firstly, a cubic smoothing spline was fitted to CO measurements. Then the point in time was calculated in which the difference between the smooth $\mathrm{CO}$ signal and the CO measurements was higher than the standard deviation of $\mathrm{CO}$ data during the first hour of the experiment. This time gave the 1- $\sigma$ cut-off point, and the method is called $\sigma$-method from here on. The analysis was done for all experiments which resulted in individual cut-off points for each experiment.

Relying on CO measurements has the disadvantage of the high noise level of $>5 \mathrm{nmol} \mathrm{mol}^{-1}$. Applying this method on $\mathrm{CH}_{4}$ measurements would be an alternative. However, implementation on $\mathrm{CH}_{4}$ did not improve the results. Moreover, $\mathrm{CH}_{4}$ measurements showed the decrease in amount fractions later than the increase in CO. The differences in the onset of the increase or decrease can be related to the underlying mechanisms in the CRDS analyzer, such as outgassing of CO followed by a dilution effect in the cavity for $\mathrm{CH}_{4}$. However, as already discussed in Sect. 3.1.1, the reason of this effect in the CRDS analyzer is unclear and needs further investigation of the materials surrounding the cavity as well as the cavity itself. 

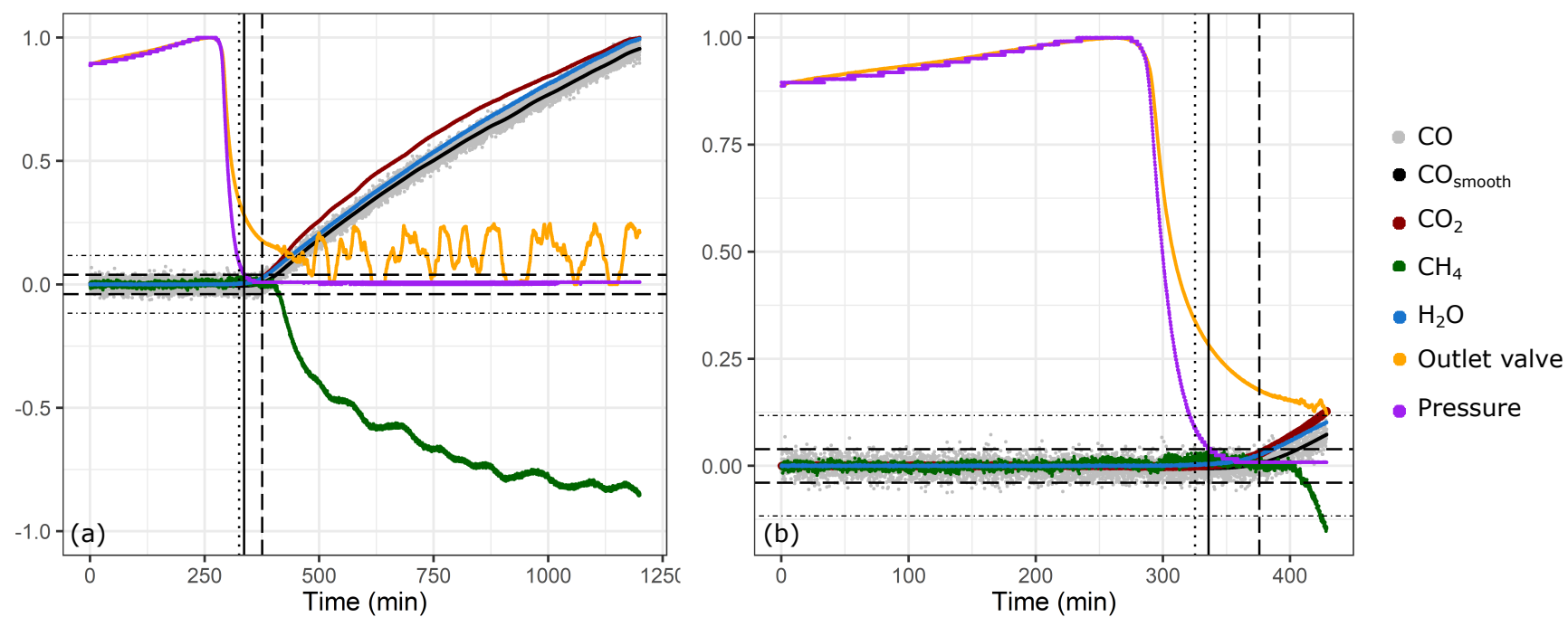

Figure S1. Normalized values of measured species, outlet valve and low pressure reading of the pressure regulator during the course of the experiment (a), and a zoom-in to first 450 minutes (b). Dot-dash lines indicate 3- $\sigma$ of the CO measurements, dashed lines indicate 1- $\sigma$, solid lines show the 25000 outlet valve cut-off point and dotted lines denotes the correlation method 


\section{S1.2 Using correlation between two measured variables}

This method is based on the correlation between the outlet valve value and the low pressure reading of the pressure regulator (Fig. S2). As the inlet pressure to the analyzer decreases, the CRDS analyzer regulates its cavity pressure through closing the outlet valve. These two variables are linked to each other as long as there is enough flow coming into the analyzer for the

5 proportional valve to regulate. Towards the end of the measurements, the correlation between the outlet valve and the pressure reading breaks, and these parameters are not physically linked anymore. In order to calculate the point in time where the measurements were still correlated, an algorithm was constructed. The algorithm is based on maximizing the coefficient of determination $\left(r^{2}\right)$. It started by calculating the linear regression parameters between the low pressure and the outlet valve data for the range where the outlet valve values are between 30000 and 40000, indicated by the blue region in the Fig. S2a. In this region these two parameters should be correlated, and sufficient flow conditions should be present. To this interval, consecutive data points were added step-by-step, and the fit was recalculated. From the calculated fits, the range which yields the highest $r^{2}$ was selected. The last data point added to this range determined the cut-off point. Then, to this range, the measurements at the beginning of the experiment was added, since under the initial conditions there was sufficient flow through the analyzer. This yielded the total range of included data for this method (Fig. S2b). Since the correlation method is based on maximizing the coefficient of determination $\left(r^{2}\right)$ for the linear fit, it is capable of capturing the linear relation, however the validity of such linear dependency is not confirmed. For instance, a time lag in the response of the outlet valve would not follow linear response. Moreover, a cut-off point based on low pressure reading would incorporate the variations in the pressure measurements, since the pressure data is measured relative to atmospheric pressure.

In order to highlight the differences between the three methods, Fig. S1 shows all cut-off points as vertical lines. Using the $\sigma$-method resulted in cut-off points later in time, whereas using the correlation approach was the most conservative approach in cutting the data. The methods excluding the 25000 cut-off point yieldeed individual cut-off points for each experiment. In Fig. S3 the distribution of the cut-off points is presented. This clearly illustrates the difference between the two approaches, whereas the value 25000 is closer to outlet valve-low pressure correlation method.

As explained in Sect. 3.1.2, we have validated that using the $\mathrm{CO}$ amount fractions did not act early enough to distinguish the instrument related effects from the desorption signal. Nevertheless, we present the results for these two other methods (Fig. S4 and Fig. S5). These results can be interpreted by using Fig. 3: the later the cut-off point, the larger will be the enrichments in the amount fractions of $\mathrm{CO}_{2}$ and $\mathrm{H}_{2} \mathrm{O}$. One-hour difference between the cut-off points from the $\sigma$-method and the outlet valve low pressure method explains the observed differences between the maximum amount fractions observed for the experiments. The difference between the methods were up to $1.1 \mu \mathrm{mol} \mathrm{mol}^{-1}$ for $\mathrm{CO}_{2}(14$ bar experiment after heating $)$ and up to 300 

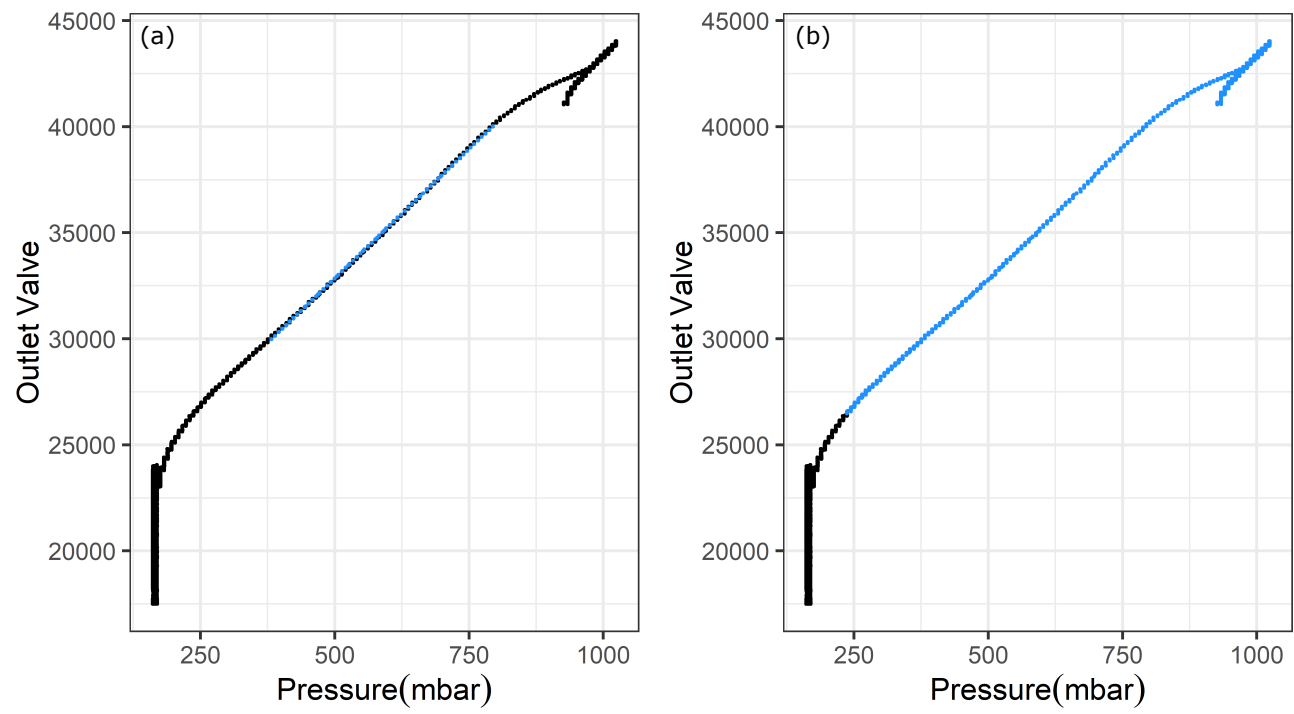

Figure S2. The initial conditions for the correlation method. Blue region indicates the included data at the beginning (a), and data to be included after the algorithm (b)

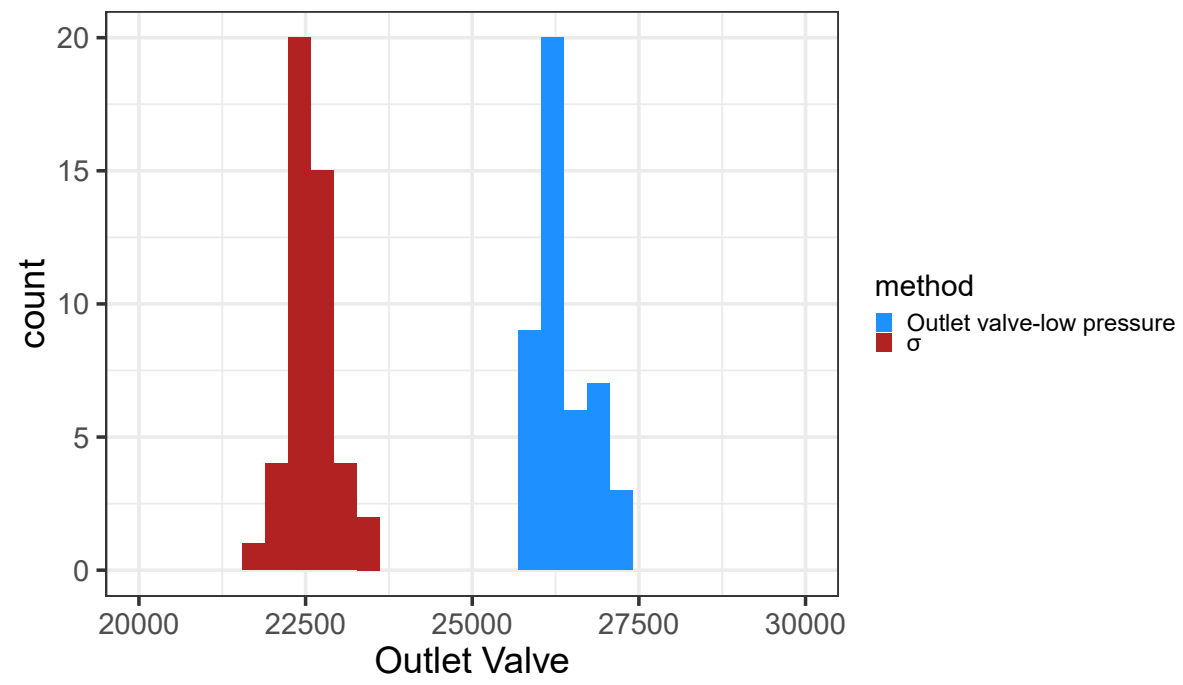

Figure S3. Distribution of the cut-off points using the two methods

$\mu$ mol mol ${ }^{-1}$ for $\mathrm{H}_{2} \mathrm{O}$ (3.5 bar experiment with mass flow meter). Using the $\sigma$-method should limit any significant enrichments of $\mathrm{CO}$, since the method is based on tracking the increase in $\mathrm{CO}$ amount fractions. However, a slight difference of $2 \mathrm{nmol} \mathrm{mol}^{-1}$ is observed for CO measurements between the two methods (Fig. S4 and Fig. S5). 
On the hand, the differences between the correlation method and the fixed outlet valve value of 25000 are small. For $\mathrm{CO}_{2}$, the differences for the aluminum cylinder were less than $0.1 \mu \mathrm{mol} \mathrm{mol}^{-1}$, whereas for the steel cylinder they were slightly bigger amounting to $1.25 \mu \mathrm{mol} \mathrm{mol}^{-1}$. The mean of the differences between the two methods were $0.3 \mathrm{nmol} \mathrm{mol}^{-1}$ and 0.1 nmol mol ${ }^{-1}$ for $\mathrm{CO}$ and $\mathrm{CH}_{4}$ respectively. For $\mathrm{H}_{2} \mathrm{O}$ a mean difference of $6 \mu \mathrm{mol} \mathrm{mol}^{-1}$ was calculated when all experi-

5 ments except the runs with mass flow meter were taken into account. The runs with MFM showed differences as high as 40 $\mu \mathrm{mol} \mathrm{mol}{ }^{-1}$. The higher increase was related to steeper slope of $\mathrm{H}_{2} \mathrm{O}$ enrichment when MFM was placed prior to the analyzer. It is important to note that the differences between the fixed outlet value of 25000 and the outlet valve pressure correlation method do not result in significant changes in the interpretation of data presented in Sect. 3.2.
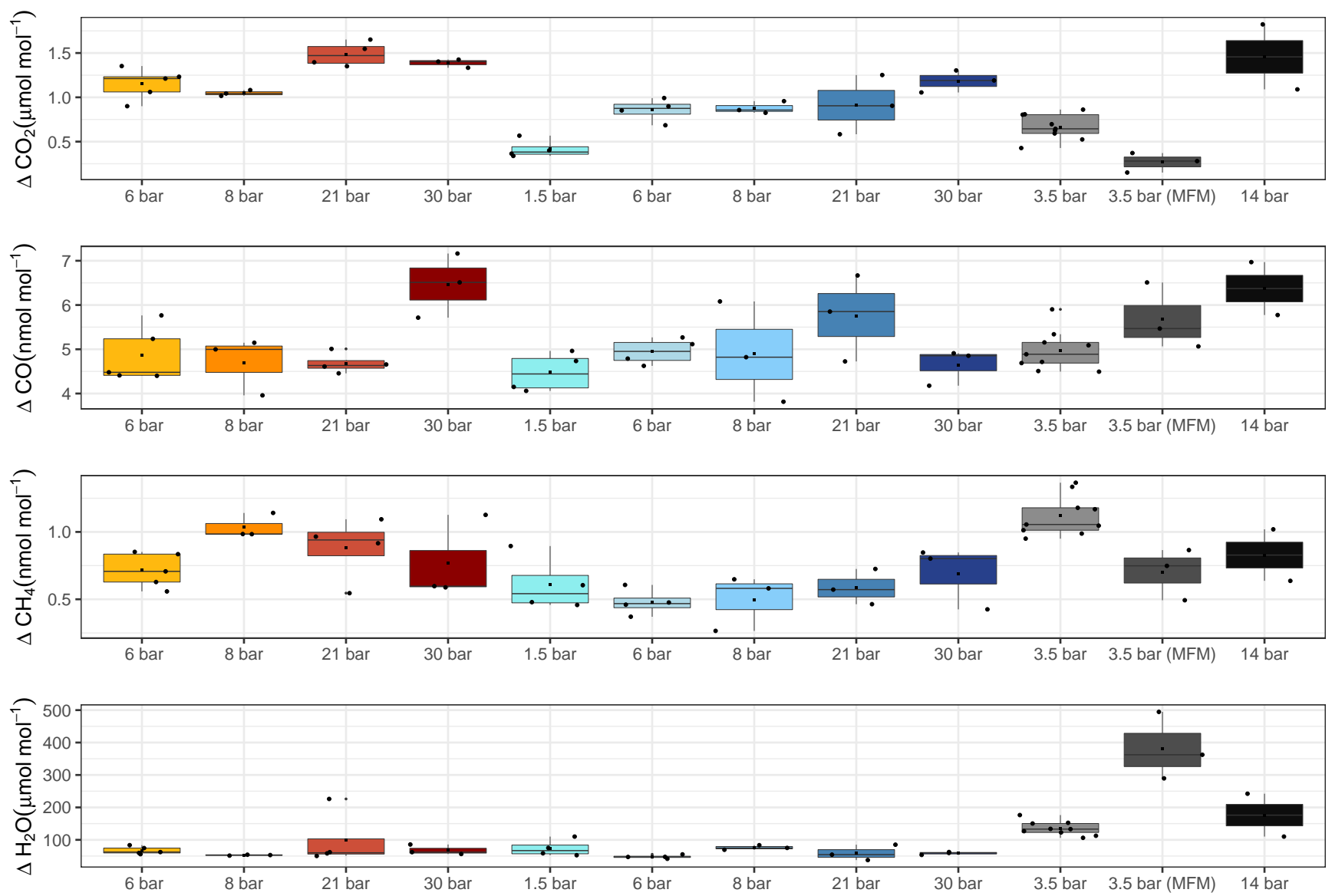

Figure S4. Box plots for all experiments for the species $\mathrm{CO}_{2}, \mathrm{CO}, \mathrm{CH}_{4}$ and $\mathrm{H}_{2} \mathrm{O}$ using the $\sigma$-method 

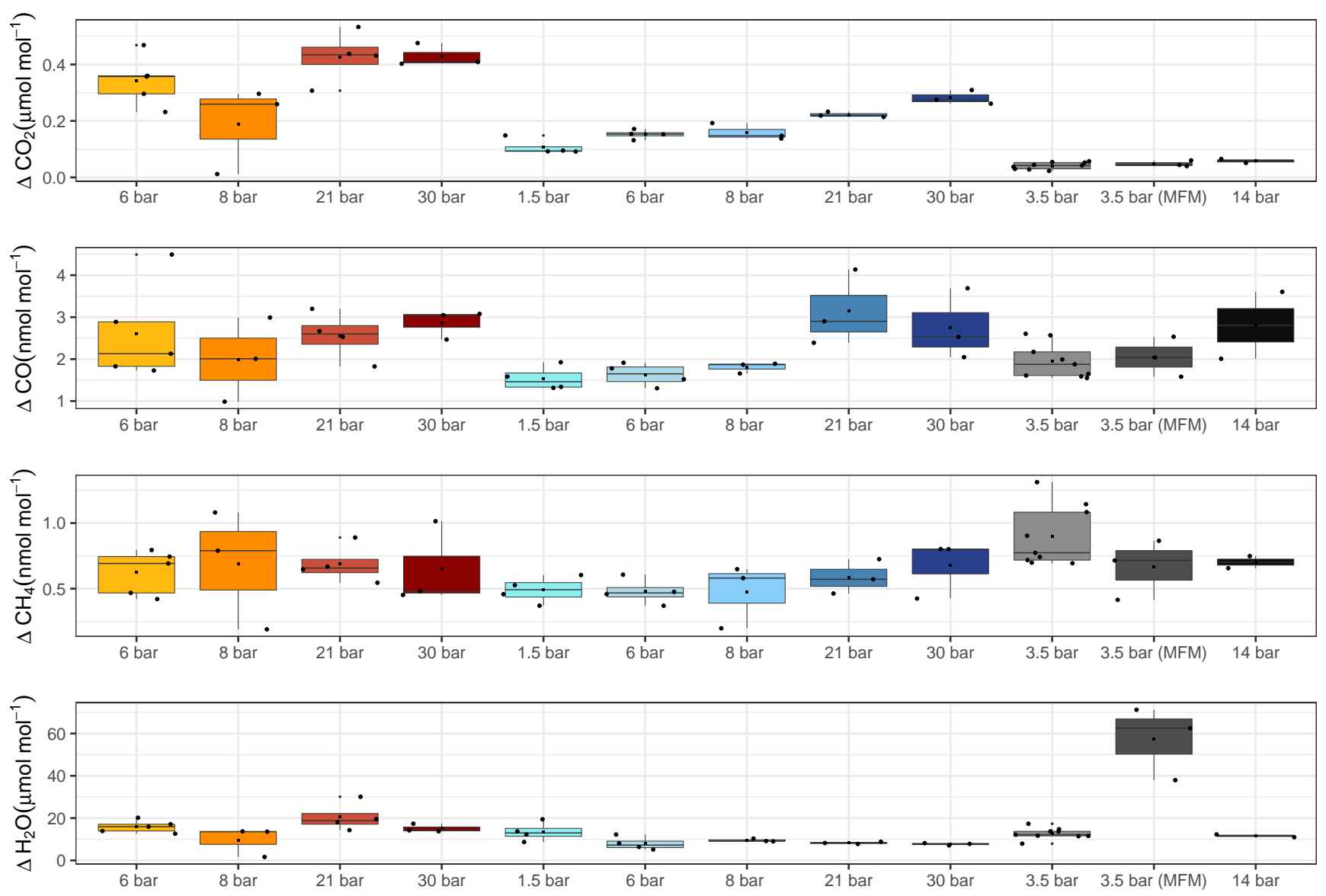

Figure S5. Box plots for all experiments for the species $\mathrm{CO}_{2}, \mathrm{CO}, \mathrm{CH}_{4}$ and $\mathrm{H}_{2} \mathrm{O}$ using the outlet valve - low pressure method 


\section{S2 Correction for the measurements}

As explained in Sect. 3.2, the measurements after heating for the aluminum cylinder showed a downwards trend in the amount fractions of $\mathrm{CO}_{2}$. Figure S6 and S7 show the evolution of this downward trend for experiments with aluminum cylinder before and after the temperature experiments. The evolution of the cavity pressure with respect to cylinder pressure and the outlet

5 valve value was similar in both cases, which supported that this behavior was related to a lag in response time of the outlet valve. However, the enrichment in the amount fractions was significantly different among the two cases. The measurements at 8 bar before heating showed a dip in the measured amount fraction in the order of $0.05 \mu \mathrm{mol} \mathrm{mol}{ }^{-1}$. This negative difference was compensated by a steep increase in the amount fraction of $\mathrm{CO}_{2}$. Whereas, for the experiments after heating the dip in the observations was as high as $0.1 \mu \mathrm{mol} \mathrm{mol}{ }^{-1}$ and the decrease was not recovered as fast as in the previous experiments. This is presumably related to the lower amount of $\mathrm{CO}_{2}$ molecules adsorbed to the surface.

A linear correction based on the cavity pressure and the observed negative deviations from the starting amount fractions was applied. The deviations were corrected with slopes varying from -1.93 to $-0.75 \mu \mathrm{mol} \mathrm{mol}^{-1} \mathrm{Torr}^{-1}$ for the experiments after the heating. Figure S8b shows the corrected amount fractions. The application of a correction function is non-trivial, since the amount fraction response at lower cavity pressures cannot be explained in its full extent by a linear function. Therefore, despite

15 the correction function, a slight decrease (in the order of $0.05 \mu \mathrm{mol} \mathrm{mol}^{-1}$ ) in the amount fractions of $\mathrm{CO}_{2}$ is still present. 

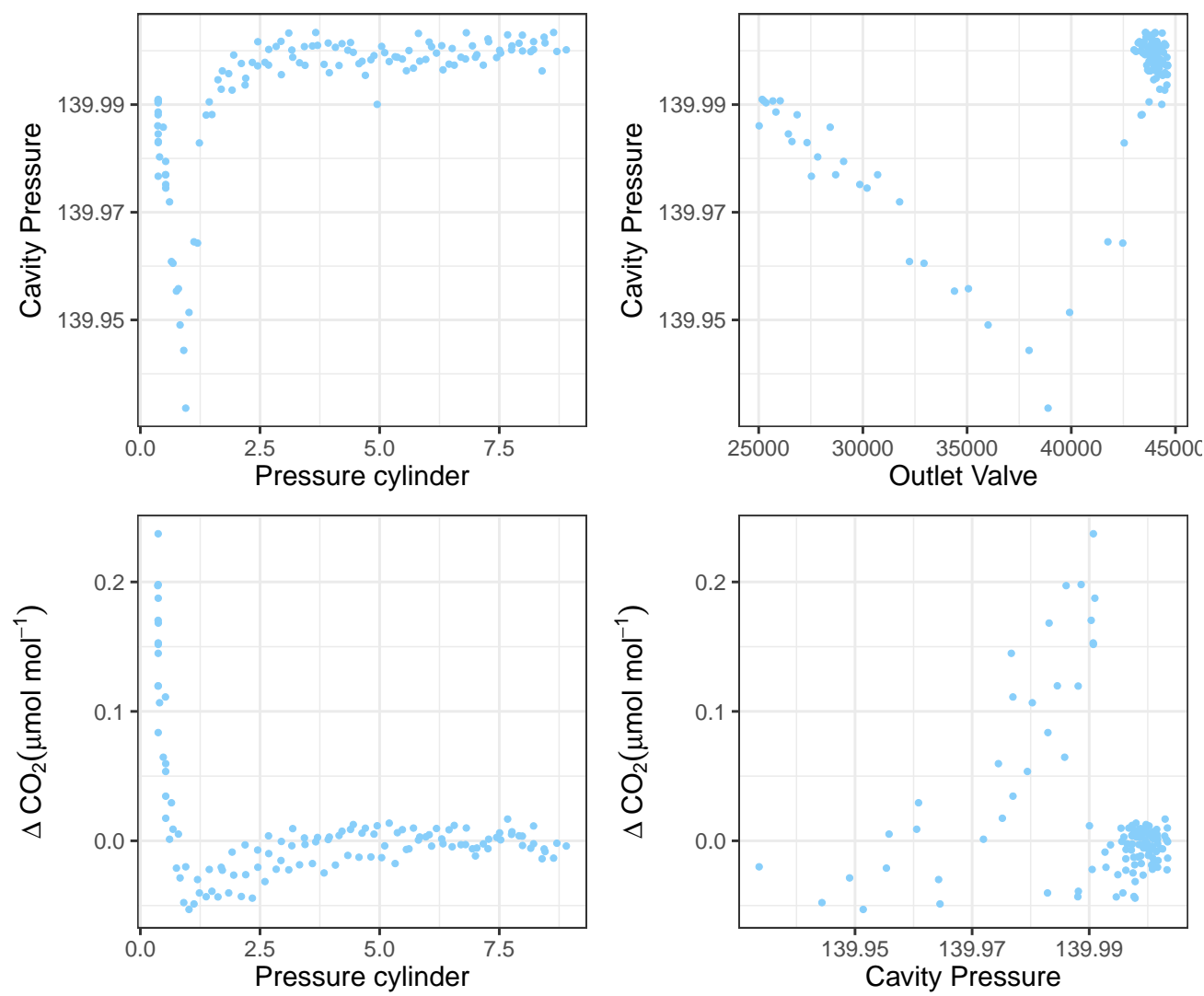

Figure S6. Response of $\mathrm{CO}_{2}$ amount fraction to changes in cavity pressure for the aluminum cylinder filled at 8 bar before heating. To make it easier to follow, same color codes are used for all experiments presented in this study. 

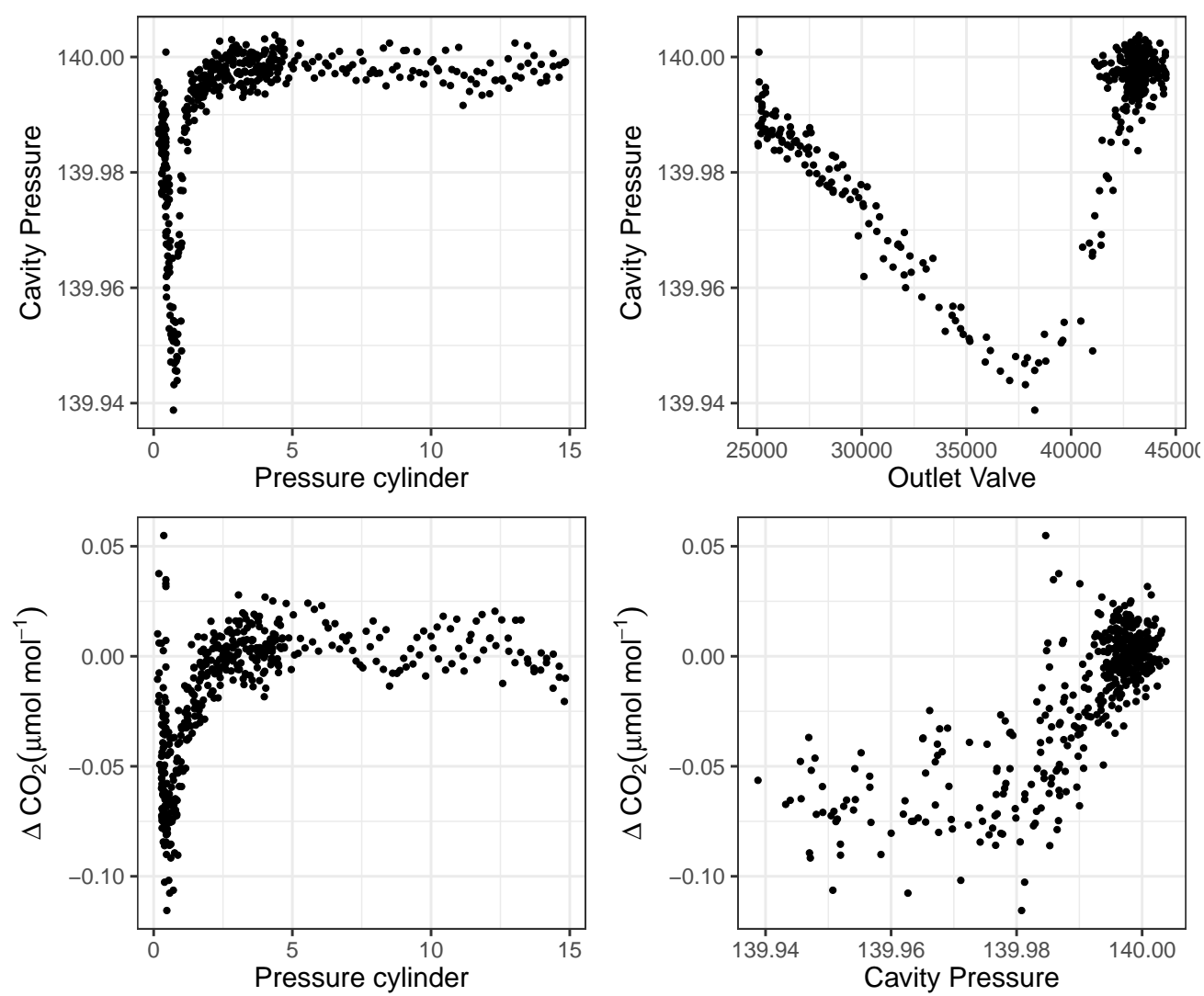

Figure S7. Response of $\mathrm{CO}_{2}$ amount fraction to changes in cavity pressure for all experiments after the heating for the aluminum cylinder. To make it easier to follow, same color codes are used for all experiments presented in this study. 

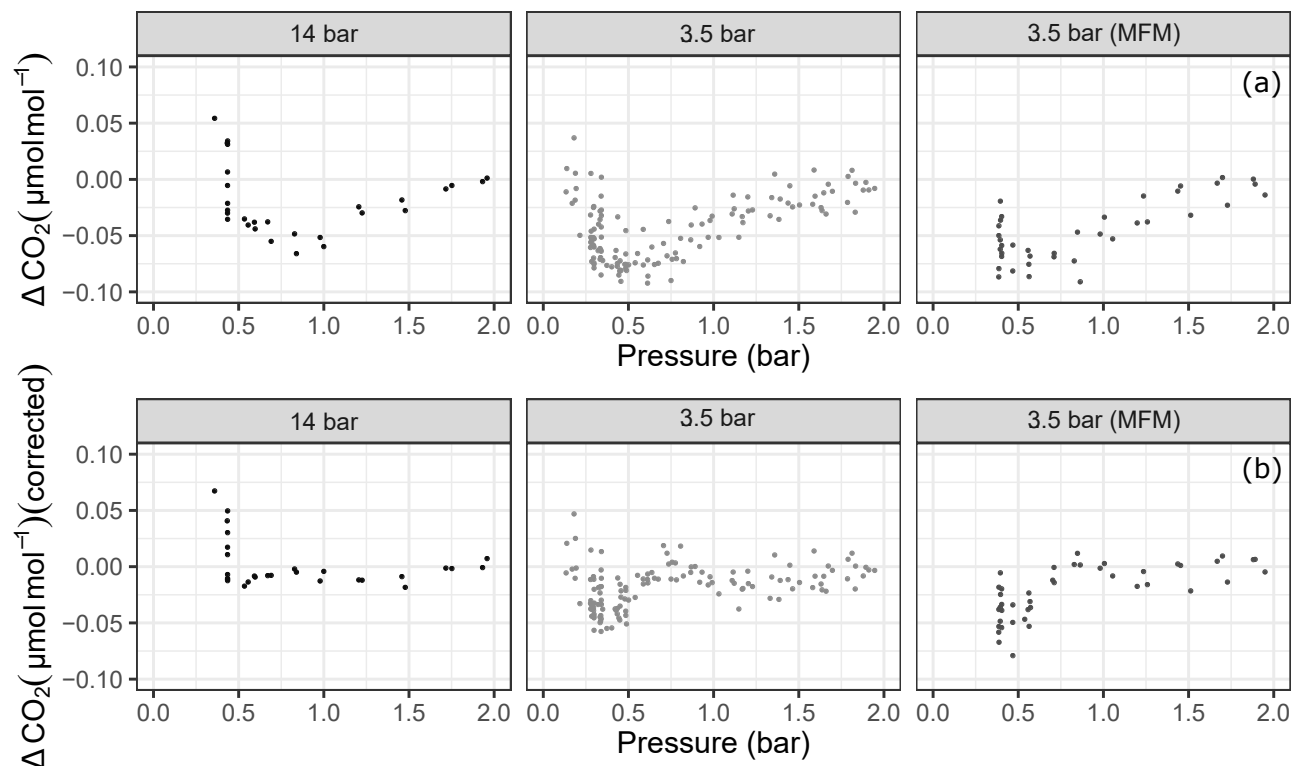

Pressure (bar)
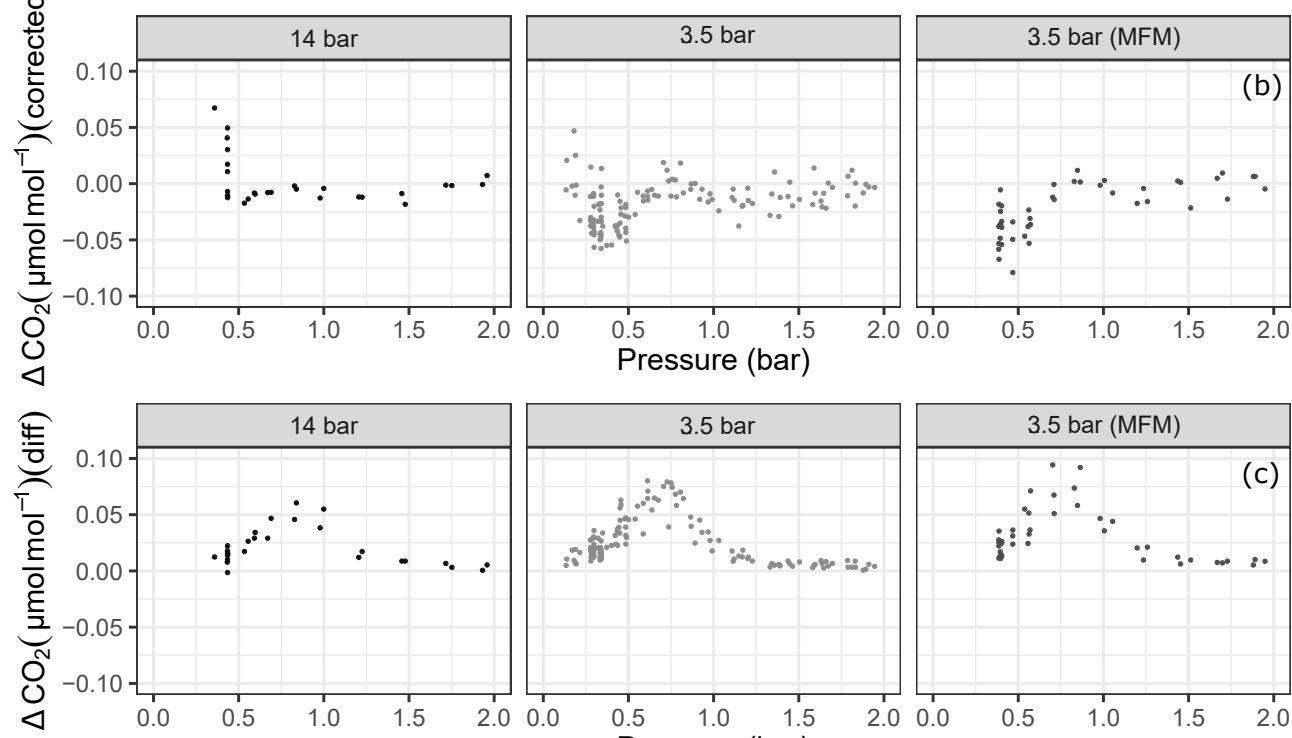

(b)
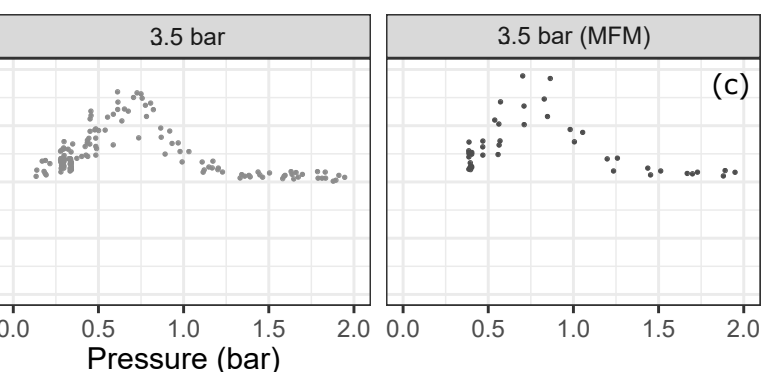

Figure S8. Zoom-in to the region in which the correction is significant. (a) Uncorrected, (b) corrected, and (c) the difference between the two values for the $\mathrm{CO}_{2}$ amount fractions. To make it easier to follow, same color codes are used for all experiments presented in this study. 


\section{S3 Langmuir adsorption/desorption model fits}

\section{S3.1 Model fits for CRDS data}

The data from this study did not support the shape of the Langmuir adsorption isotherm as observed in the previous studies (Leuenberger et al., 2015; Brewer et al., 2018; Schibig et al., 2018). The onset of the surface effects was not observed until sub

5 atmospheric pressures for the cylinders tested in this study.

In order to investigate whether the observed amount fraction changes can be explained by the Langmuir adsorption isotherm for monolayer coverage, we used a modified version of the Eqn. 5 from Leuenberger et al. (2015):

$C O_{2, \text { measured }}-\mathrm{CO}_{2, \text { initial }}+C \mathrm{O}_{2, a d s}=\Delta C \mathrm{O}_{2}=C O_{2, a d s} \cdot\left(\frac{K \cdot\left(P-P_{0}\right)}{1+K \cdot P}+\left(1+K \cdot P_{0}\right) \cdot \ln \left(\frac{P_{0} \cdot(1+K \cdot P)}{P \cdot\left(1+K \cdot P_{0}\right)}\right)\right)$

Where, $\mathrm{CO}_{2, \text { initial }}+\mathrm{CO}_{2, \text { ads }}$ is the mean of the measured amount fractions during the first hour for each experiment.

Therefore, for $\mathrm{P}$ close to $\mathrm{P}_{0}$, the left side of Eqn. $\mathrm{S} 1$ will be close to zero and it increases with lower pressures. The left term on the right hand side of Eqn. $\mathrm{S} 1$ is always negative and the right term always positive. Increasing $\mathrm{K}$ or $\mathrm{CO}_{2, a d s}$ values increases the left term. Yet $K$ increase is less pronounced compared to $C O_{2, a d s}$ change. $K$ determines the curvature whereas $C O_{2, a d s}$ just stretch or compress the values.

In order to find the best possible fit, we have used R's inbuilt opt im function with the setting Limited-memory Broyden-

15 Fletcher-Goldfarb-Shanno (BFGS) algorithm. Upper and lower bounds were set for each unknown $\left(\mathrm{CO}_{2, a d s}\right.$ and $\left.\mathrm{K}\right)$ and the algorithm was run to minimize the sum of squared differences between the measured amount fractions and the modelled amount fractions. For $C O_{2, a d s}$ lower and upper boundaries were set as $0.001 \mu \mathrm{mol} \mathrm{mol}^{-1}$ and $15 \mu \mathrm{mol} \mathrm{mol}{ }^{-1}$. We have set the first guess values for the algorithm to the lower boundaries. In Fig. S9, we show the theoretical isotherms together with our experimental data. The purple points show measurement data from the 30 bar experiments of the aluminum cylinder, and the black lines show the Langmuir monolayer fit to the measurements with $\mathrm{K}$ values of $0.001 \mathrm{bar}^{-1}$ and $1 \mathrm{bar}^{-1}$ denoted by the solid and dashed lines, respectively. Fig.S9b shows a zoom-in to the region where the pressure in the cylinder is less than 3 bar. In order to find a better fit to the experimental data, we have further increased the upper limit of the $K$ value up to 500 bar $^{-1}$ (Table S1). At higher K values, the modelled curve fits better to the onset of the increasing amount fractions. 


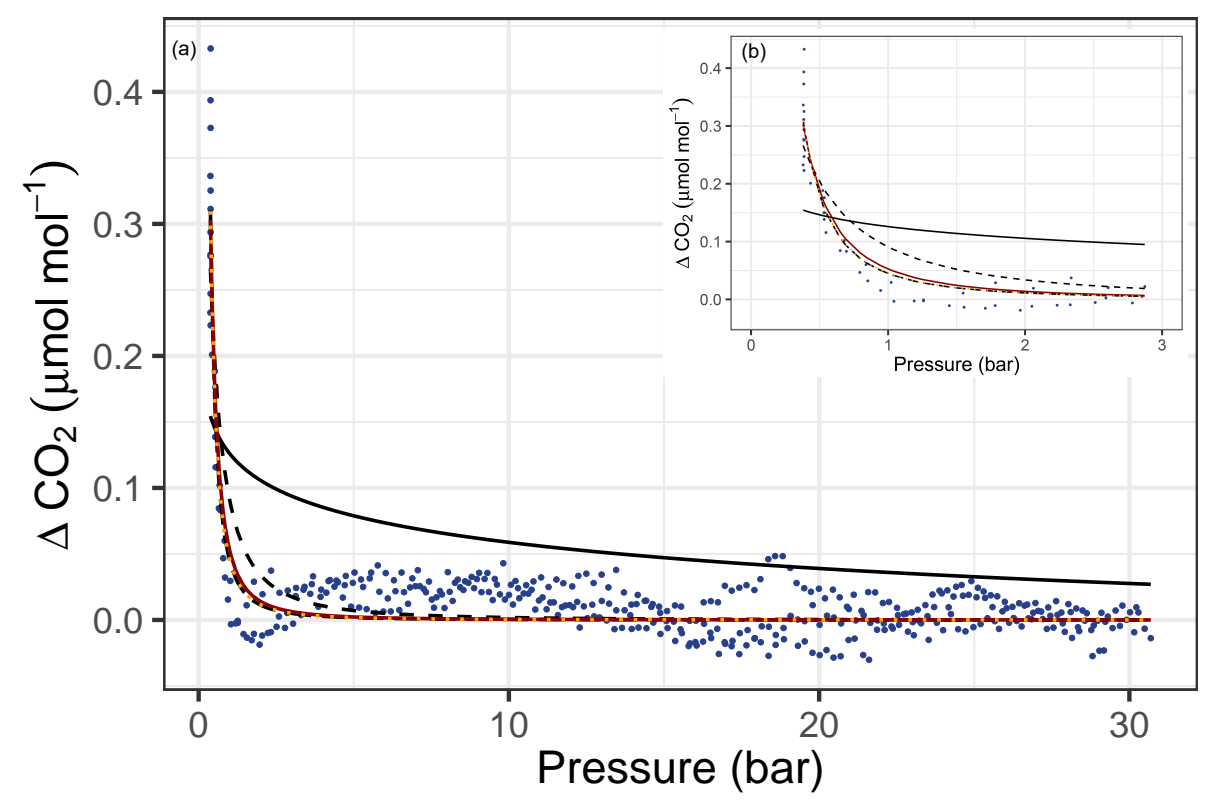

Figure S9. (a)Measured and modelled amount fractions of $\mathrm{CO}_{2}$ for the aluminum cylinder filled to 30 bar. Purple points show measured data, black solid lines show the fit with $\mathrm{K}=0.001 \mathrm{bar}^{-1}$, black dashed lines show the fit $\mathrm{K}=1 \mathrm{bar}^{-1}$, dark red lines show the fit with $\mathrm{K}=10$ $\mathrm{bar}^{-1}$, and orange dotted lines show the fit $\mathrm{K}=100 \mathrm{bar}^{-1}$, and black long dashed lines show the fit $\mathrm{K}=379 \mathrm{bar}^{-1}$ (b) Zoom-in to the region where the cylinder pressure is less than 3 bar.

Table S1. Model parameters for Langmuir adsorption isotherm for CRDS data

\begin{tabular}{lc}
\hline$K\left(\mathrm{bar}^{-1}\right)^{[1]}$ & $\left.C O_{2, a d s}(\mu \mathrm{mol} \mathrm{mol})^{-1}\right)^{[2]}$ \\
\hline 0.001 & 0.029 \\
1 & 0.015 \\
10 & 0.038 \\
100 & 0.301 \\
$379[3]$ & 1.116 \\
\hline
\end{tabular}

[1] Upper boundary for $\mathrm{K}$ is increased from $0.001 \mathrm{bar}^{-1}$ to $500 \mathrm{bar}^{-1}$ stepwise for each solution.

[2] Lower and upper boundaries for $C_{2}$,ads $0.001 \mu \mathrm{mol} \mathrm{mol}^{-1}$ and $15 \mu \mathrm{mol} \mathrm{mol}{ }^{-1}$

[3] The best fit was not limited by the boundary conditions 
The optimization algorithm was run similarly to Sec. S3.1. The experimental results supported the Langmuir model, however, the enrichments occurred in the region where the pressure correction function required extrapolation. Therefore, these data should be interpreted carefully.

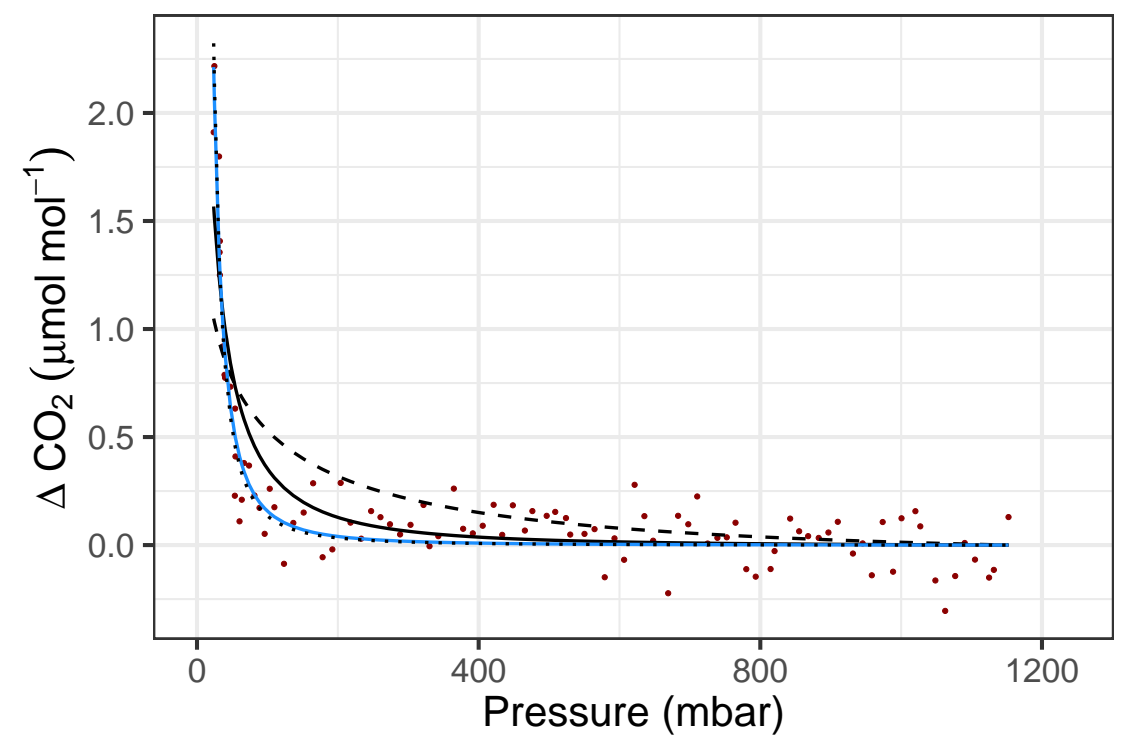

Figure S10. : Measured and modelled amount fractions of CO2 for the aluminum cylinder from the QCLAS setup. Red points show measured data, black dashed lines show the modelled fit with $\mathrm{K}=0.001 \mathrm{bar}^{-1}$, black lines show the modelled fit with $\mathrm{K}=0.01$ bar ${ }^{-1}$, blue lines show the modelled fit with $\mathrm{K}=0.152 \mathrm{bar}^{-1}$ and black dotted lines show the modelled fit with $\mathrm{K}=1 \mathrm{bar}^{-1}$

Table S2. Model parameters for Langmuir adsorption isotherm for QCLAS data

\begin{tabular}{lc}
\hline$K\left(\mathrm{bar}^{-1}\right)^{[1]}$ & $\left.C O_{2, a d s}(\mu \mathrm{mol} \mathrm{mol})^{-1}\right)^{[2]}$ \\
\hline 0.001 & 0.185 \\
0.01 & 0.149 \\
$0.152^{[3]}$ & 0.454 \\
1 & 2.387 \\
\hline
\end{tabular}

[1] Upper boundary for $\mathrm{K}$ is increased from $0.001 \mathrm{bar}^{-1}$ to $1 \mathrm{bar}^{-1}$ stepwise for each solution.

[2] Lower and upper boundaries for $C O_{2, a d s} 0.001 \mu \mathrm{mol} \mathrm{mol}^{-1}$ and $15 \mu \mathrm{mol} \mathrm{mol}{ }^{-1}$

[3] The best fit was not limited by the boundary conditions 


\section{References}

Brewer, P. J., Brown, R. J. C., Resner, K. V., Hill-Pearce, R. E., Worton, D. R., Allen, N. D. C., Blakley, K. C., Benucci, D., and Ellison, M. R.: Influence of Pressure on the Composition of Gaseous Reference Materials, Analytical Chemistry, 90, 3490-3495, https://doi.org/10.1021/acs.analchem.7b05309, https://doi.org/10.1021/acs.analchem.7b05309, pMID: $29381338,2018$.

5 Hawkins, E. and Sutton, R.: Time of emergence of climate signals, Geophysical Research Letters, 39, L01702, https://doi.org/10.1029/2011g1050087, 2012.

Leuenberger, M. C., Schibig, M. F., and Nyfeler, P.: Gas adsorption and desorption effects on cylinders and their importance for long-term gas records, Atmospheric Measurement Techniques, 8, 5289-5299, https://doi.org/10.5194/amt-8-5289-2015, https://www.atmos-meas-tech. net/8/5289/2015/, 2015.

10 Schibig, M. F., Kitzis, D., and Tans, P. P.: Experiments with $\mathrm{CO}_{2}$-in-air reference gases in high-pressure aluminum cylinders, Atmospheric Measurement Techniques, 11, 5565-5586, https://doi.org/10.5194/amt-11-5565-2018, https://www.atmos-meas-tech.net/11/5565/2018/, 2018. 